\title{
Professionelle Personalentwicklung in einer heterogenen Trägerlandschaft der Kindertagesbetreuung
}

\author{
Petra Strehmel \\ Department Soziale Arbeit, Hochschule für Angewandte Wissenschaften Hamburg
}

\begin{abstract}
Zusammenfassung: Personalentwicklung in Kindertageseinrichtungen ist eine zentrale Voraussetzung zur Sicherung und Weiterentwicklung der pädagogischen Qualität. Die Zuständigen für das Personal bei den Trägern handeln im Kontext ihrer jeweiligen Organisationen, die im System der Kindertagesbetreuung u. a. hinsichtlich ihrer Größe oder der Haupt- oder Ehrenamtlichkeit der Trägerverantwortlichen sehr heterogen aufgestellt sind. Daher stellt sich die Frage, unter welchen organisationalen Voraussetzungen die Träger eine professionelle Personalarbeit leisten. Analysen des Forschungsstandes, der Trägerlandschaft sowie der Befunde einer qualitativen multiplen Fallstudie kommen zu dem Schluss, dass auch kleine und ehrenamtlich geführte Träger die kontinuierliche Weiterentwicklung des Personals professionell gestalten können, wenn sie das Unterstützungssystem aus Dachverbänden nutzen.
\end{abstract}

Schlüsselwörter: Personalentwicklung, Arbeitssystem, Träger, Wohlfahrtsverbände, Ehrenamtlichkeit

Professional Staff Development in a Diverse Landscape of Provider Organizations of Early Child Care Centers

Abstract: Staff development in early childhood centers is an important basis for ensuring and promoting pedagogical quality. Managers in charge of the staff act in the context of their entire organizations, which may have a very diverse structure within the overall system of early childhood education, for example, regarding the size of the organizations or the form of management (voluntary or professional). This leads to the question under which conditions managers can best carry out their duties of professional human resource management in early childhood provider organizations. Analyses of the state of research, the present landscape of provider organisations in Germany, and of results from a qualitative multiple case study conclude that even small and voluntarily managed provider organisations are able to promote the continuous professional development of the staff, if they tap into the support system of umbrella associations.

Keywords: staff development, working system, provider organisation, welfare associations, voluntary work

Pädagogische Qualität in Kindertageseinrichtungen entwickelt sich in einem kompetenten System durch das $\mathrm{Zu}$ sammenspiel zwischen den Akteurinnen und Akteuren auf verschiedenen Ebenen (EU, 2011). Durch die Steuerung des Systems soll dafür gesorgt werden, dass „Einrichtungen und Fachkräften Rahmenbedingungen und Unterstützungsstrukturen zur Verfügung stehen, die die Bereitstellung eines qualitativ hochwertigen Angebots frühkindlicher Bildung, Erziehung und Betreuung ermöglichen" (BMFSFJ/JFMK, 2016, S.75). Eine wichtige Voraussetzung für die Steuerung ist das Wissen um Strukturen, Arbeitsweisen und Formen des Zusammenwirkens zwischen den Beteiligten auf verschiedenen Ebenen des Systems. Dies betrifft auch die Personalentwicklung, denn pädagogische Qualität erfordert u.a. eine hohe Qualifikation des Personals sowie dessen kontinuierliche professionelle Weiterentwicklung (EU, 2021).
Die Verantwortung für die Personalentwicklung liegt formal beim Träger als Arbeitgeber. Im Hinblick auf die Steuerung des Systems wird konstatiert: „Mit der hohen Verantwortung der Träger von Kindertageseinrichtungen geht ein Anspruch an die Professionalität des Trägers einher. Es wird ein hoher Professionalisierungsbedarf insbesondere kleiner Träger angenommen bzw. die Einbindung kleiner Träger in professionelle Strukturen als notwendig erachtet" (BMFSFJ/ JFMK, 2016, S.53). Geiger (2019, S.98) sieht auf der Grundlage einer bundesweiten Befragung von Kindertageseinrichtungen ebenfalls ein Professionalisierungsbedarf bei den Trägern und stellt mehr Fachkompetenz für Personalentwicklung bei größeren Trägern fest. Klug und Kratzmann (2016) gehen davon aus, dass nur größere und hauptamtlich geführte Träger die Aufgaben der Personalentwicklung in Kindertageseinrichtungen in ausreichender Qualität erfüllen können. Sie fordern „die Existenz von Kleinstträgern zu beenden und größere professionell geführte Einheiten zu 
schaffen" (S.165). Eine ideale Größe seien Träger mit 10-20 Kitas (Klug \& Kratzmann, 2016). Diese Aussagen werden kaum empirisch begründet und es bleibt unklar, was eigentlich mit „Professionalität" gemeint ist. Und inwieweit passen diese Vorstellungen zu den Trägerstrukturen im System der Kindertagesbetreuung?

Professionalität kann verstanden werden als ein Handeln auf der Basis wissenschaftlicher Erkenntnisse und fachspezifischer Kompetenzen, welches sich durch spezifisches Wissen, Können und eine reflektierte Haltung von laienhaften Verhaltensweisen unterscheidet (Langer, 2018). Professionelle Personalentwicklung in Kindertageseinrichtungen müsste sich demnach an Kriterien orientieren, die durch Forschungsergebnisse über Effekte bestimmter Instrumente der Personalentwicklung begründet sind.

Die Trägerlandschaft in der Kindertagesbetreuung ist indessen sehr heterogen und es stellt sich die Frage, wie unterschiedlich strukturierte Träger dem Anspruch an Professionalität in der Personalentwicklung gerecht werden. Welche Schlussfolgerungen ergeben sich daraus für die Steuerung des Systems?

Zunächst werden Kriterien professioneller Personalentwicklung in Kitas herausgearbeitet. Anschließend wird die Trägerlandschaft der Kindertagesbetreuung im Hinblick auf ihre Potenziale für Professionalität analysiert. Daran anknüpfend werden Ergebnisse einer qualitativen multiplen Fallstudie dargelegt, welche die Strategien und Maßnahmen der Personalentwicklung bei unterschiedlich strukturierten Trägern untersucht hat.

\section{Personalentwicklung in Kindertageseinrichtungen: Aufgaben und Forschungsstand}

Personalentwicklung sei definiert durch Strategien und Maßnahmen, mit denen Lern- und Entwicklungsprozesse der Akteurinnen und Akteure in Organisationen gezielt angestoßen und begleitet werden, um die professionelle und persönliche Weiterentwicklung des Personals zu ermöglichen und voranzutreiben (Strehmel \& Overmann, 2018). Ein Großteil des Lernens in Organisationen findet im Prozess der Arbeit selbst statt (Zapf, 2016, S.234) und kann u. a. durch lernförderliche Aufgabenprofile und Reflexionsmöglichkeiten gefördert werden (Sonntag \& Schaper, 2016, S.396). In diesem Sinne ist der Begriff der Personalentwicklung weit gefasst: Personalentwicklung beginnt bereits bei der Einarbeitung von Mitarbeiterinnen und Mitarbeitern und wird durch Maßnahmen der Bereitstellung von Lerngelegenheiten für das Kita-Personal etwa durch Teamtage, Fachberatung, Coaching oder Supervision sowie kon- tinuierliche Fort- und Weiterbildung im Verlauf der Berufsbiografie fortgesetzt (EU, 2021, S. 87).

Wer nimmt Aufgaben der Personalentwicklung im System der Kindertagesbetreuung wahr? Im Rahmen der „Nationalen Qualitätsinitiative“ (NQI) wurden 2318 Rechtsträger von Kindertageseinrichtungen nach ihren Tätigkeitsbereichen befragt (Kalicki, 2003) und dabei das Personalmanagement als eine der zentralen Aufgaben identifiziert. Als Qualitätskriterien wurden die Regelung von Personalentwicklungsmaßnahmen, die Herstellung von Transparenz über das jeweilige Personalentwicklungskonzept, die Abstimmung der Fortbildungsbedarfe sowie die Unterstützung der Teamentwicklung benannt (Oberhuemer, 2003, S.60f.). Geiger (2019) kam in ihrer bundesweiten Befragung von 1431 Kitas zu dem Schluss, dass die Kita-Leitungen ,in fast allen Kindertageseinrichtungen in Aufgaben der Personalentwicklung einbezogen und nicht selten überwiegend oder voll dafür verantwortlich" seien (Geiger, 2019, S. 63). Aufgaben der Personalentwicklung werden demnach häufig von den Trägern an die Kita-Leitungen delegiert bzw. in Arbeitsteilung mit den Leitungskräften wahrgenommen.

Im Hinblick auf die Wirksamkeit von Personalentwicklungsmaßnahmen haben sich nach internationalen Metaanalysen bestimmte Formate von Fort- und Weiterbildungen sowie Lernprozesse im Prozess der Arbeit - beispielsweise unterstützt durch Coaching, Mentoring oder kollegiale Beratung - als effektiv erwiesen (OECD, 2018, S.79). Die Maßnahmen erwiesen sich insbesondere dann als wirksam, wenn sie an Qualitätsprofilen für die pädagogische Arbeit orientiert waren, langfristig angelegt und individuell zugeschnitten waren, wenn sie nah am Arbeitsplatz stattfanden und mit Feedback-Komponenten sowie Gelegenheiten zur Reflexion (z.B. im Team) verknüpft waren (Eurofound, 2015, S.58; vgl. auch Egert, Eckhard \& Fukkink, 2017). Fortbildungen und weitere Maßnahmen zur kontinuierlichen professionellen Weiterentwicklung im Prozess der Arbeit der pädagogischen Fachkräfte erwiesen sich als ,"most consistent predictor of quality staffchild-interactions, and also has direct links to child development and learning" (OECD, 2018, S.79).

\section{Schlussfolgerungen: Kriterien professioneller Personal- entwicklung in Kitas}

Aus den bisher vorliegenden Befunden zu Maßnahmen lassen sich erste vorläufige Kriterien für eine professionelle Personalentwicklung im Bereich der Kindertagesbetreuung benennen. Personalentwicklung kann demnach gemessen werden an 
- dem Zugang der Fach- und Leitungskräfte zu individuell passenden Fort- und Weiterbildungsmaßnahmen,

- der Unterstützung professioneller Lernprozesse im Prozess der Arbeit, zum Beispiel durch Feedback und kollegiale Beratung im Team,

- die Unterstützung und Vertiefung professionellen Lernens durch Fachberatung, Coaching und Supervision.

Es gibt Hinweise, dass die Umsetzung der Personalentwicklungsaufgaben zu einem großen Teil bei den Leitungskräften liegt, während die Träger den Rahmen setzen, informieren und unterstützen. Dabei stellt sich die Frage, inwieweit es unterschiedlich strukturierten Trägern gelingt, den Einrichtungen die notwendigen Rahmenbedingungen und Ressourcen zur Personalentwicklung zur Verfügung zu stellen.

\section{Der organisationale Kontext: Träger im System der Kindertagesbetreuung}

Träger sind Organisationen, die den Betrieb von Kindertageseinrichtungen auf Grundlage der gesetzlichen Bestimmungen und eigenen Wertvorstellungen organisieren. Sie sind Arbeitgeber für das Personal, bewirtschaften die Ressourcen (Finanzen, Gebäude) und verantworten die Einhaltung aller relevanten gesetzlichen Bestimmungen. Träger beeinflussen damit auch die pädagogische Qualität (Peucker, Pluto \& Santen, 2017, S.29f.).

Der größere Teil der Kindertageseinrichtungen wird von freien Trägern betrieben. Dazu gehören frei-gemeinnützige nicht-konfessionelle oder konfessionelle (kirchliche) Träger sowie privat-gewerbliche Unternehmen. Daneben betreiben Kommunen als öffentliche Träger der Kinder- und Jugendhilfe eigene Kindertageseinrichtungen. Innerhalb dieser Gruppen unterscheiden sich Trägerorganisationen erheblich voneinander - etwa hinsichtlich ihres Angebotsspektrums, ihrer Größe, Rechtsform und Organisationsstruktur sowie hinsichtlich des Status und der Qualifikationen der Personalverantwortlichen. In Kindertageseinrichtungen in der Trägerschaft von kleineren Kommunen, Vereinen, Kirchengemeinden oder Sozialunternehmen stehen in der Regel Ehrenamtliche an der Spitze: ehrenamtliche Bürgermeisterinnen bzw. Bürgermeister, Vereinsvorstände oder kirchliche Gremien. Größere Träger beschäftigen häufig hauptamtliche Geschäftsführungen oder firmieren als gGmbHs mit professioneller Leitung.

Die meisten Kita-Träger sind Mitglieder in den Spitzenverbänden der Wohlfahrtspflege oder anderen Dachverbänden, welche die Organisationen durch Beratung und
Fortbildungsangebote unterstützen und deren fachpolitische Vertretung übernehmen (vgl. Falkenhagen, Frauendorf \& Bender, 2017).

Tabelle 1 gibt einen Überblick über die Trägerlandschaft im Arbeitsfeld der Kindertagesbetreuung in Deutschland (Statistisches Bundesamt, 2020, S.17f.)

Kindertageseinrichtungen der öffentlichen Kinder- und Jugendhilfeträger, frei-gemeinnützige nicht-konfessionelle Einrichtungen und konfessionelle Einrichtungen machen jeweils ein knappes Drittel aus, privat-gewerbliche Träger bundesweit unter $3 \%$. Mehr als jede sechste Kita (15\%) gehört keinem der Spitzenverbände der Wohlfahrtspflege an. Mehr als jede vierte Kindertageseinrichtungen (26.2\%) wird von einem Verein getragen (Statistisches Bundesamt, 2020, S.17f.; eigene Berechnung). Eingetragene Vereine werden von ehrenamtlichen Vorständen geführt, die nicht notwendig Fachexpertise für Frühe Bildung, Leitung und Management mitbringen. In einer Studie von Beher, Krim-

Tabelle 1. Verteilung der Träger von Kindertageseinrichtungen

\begin{tabular}{|c|c|c|c|}
\hline & Träger & Anzahl & $\%$ \\
\hline \multicolumn{4}{|c|}{ ÖFFENTLICHE TRÄGER } \\
\hline & Örtliche Träger & 5507 & \\
\hline & Überörtliche Träger und Land & 82 & \\
\hline & Gemeinden ohne Jugendamt & 13295 & \\
\hline & Zusammen & 18884 & 32,7 \\
\hline \multicolumn{4}{|l|}{ FREIE TRÄGER } \\
\hline \multicolumn{4}{|c|}{ Gemeinnützige Träger } \\
\hline \multirow{5}{*}{$\begin{array}{l}\text { Nicht- } \\
\text { konfessionelle } \\
\text { Träger }\end{array}$} & Arbeiterwohlfahrt (AWO) & 2607 & \\
\hline & Paritätischer Wohlfahrtsverband & 5282 & \\
\hline & DRK & 1714 & \\
\hline & Andere & 8676 & \\
\hline & Zusammen & 18279 & 31,7 \\
\hline \multirow{5}{*}{$\begin{array}{l}\text { Konfessionelle } \\
\text { Träger }\end{array}$} & Evangelische Kirche & 9107 & \\
\hline & Katholische Kirche & 9413 & \\
\hline & $\begin{array}{l}\text { Zentralwohlfahrtsstelle } \\
\text { der Juden }\end{array}$ & 20 & \\
\hline & Andere & 218 & \\
\hline & Zusammen & 18758 & 32,6 \\
\hline \multicolumn{2}{|c|}{ Freie Träger insgesamt } & 37037 & \\
\hline \multicolumn{4}{|c|}{ Nicht-gemeinnützige Träger } \\
\hline & Betriebliche Einrichtungen & 153 & \\
\hline & privat-gewerbliche Träger & 696 & \\
\hline & Andere & 824 & \\
\hline & Zusammen & 1673 & 2,9 \\
\hline & Insgesamt & 57594 & \\
\hline
\end{tabular}

Anmerkungen: Quelle: Eigene Berechnungen (nach Daten des Statistischen Bundesamts 2020, S.17f.) 
mer, Rauschenbach \& Zimmer (2008) verfügte die Mehrzahl ehrenamtlicher Führungskräfte über einen akademischen Abschluss, hatte bereits ehrenamtliche Arbeit kennengelernt und Verantwortung übernommen.

In der AQUA-Studie (,Arbeitsplatz und Qualität in Kitas") wurden u.a. bundesweit 1524 Kita-Träger befragt (Schreyer et al., 2014, S.16). Der Großteil der Kita-Träger (85\%) betrieb bis zu zehn Kitas, weitere $10 \%$ bis zu 25 Einrichtungen (Schreyer et al., 2014, S.156). Ein knappes Viertel der Träger-Vertreterinnen und -vertreter gab an, ehrenamtlich zu arbeiten: 7\% im kommunalen Bereich, aber $44 \%$ bei den freien Trägern und etwas mehr als ein Viertel bei den kirchlichen Trägern (Schreyer et al., 2014, S.151).

Die Trägerlandschaft erweist sich damit als sehr heterogen. Typisch sind kleinere Träger mit bis zu zehn Einrichtungen. Ehrenamtlich geführte Träger stellen einen bedeutsamen Anteil der Kita-Plätze in Deutschland zur Verfügung.

Hinsichtlich der Instrumente der Personalentwicklung wiesen in der AQUA-Studie freie Träger häufiger als kommunale Träger Merkmale eines professionellen Personalmanagements auf. Mehr als drei Viertel der kirchlichen Träger verfügte über Stellenbeschreibungen für Kita-Leitungen gegenüber 52\% der kommunalen Träger (Schreyer et al., 2014, S.168). Auch im Hinblick auf Einarbeitungskonzepte, Leitbilder, die Zertifizierung des Trägers durch ein Gütesiegel oder Personalentwicklungsmaßnahmen für Kita-Leitungskräfte waren die freien gegenüber den kommunalen Trägern besser aufgestellt (Schreyer et al., 2014, S.169ff.).

In der Studie von Geiger (2019) schätzten über $80 \%$ der befragten Kitas Teamsitzungen als wichtigstes Instrument ein. Weitere als sehr bedeutsam eingeschätzte Maßnahmen waren die Einarbeitung neuer Kräfte, Fort- und Weiterbildung, Team- bzw. Klausurtage, Personal- und Mitarbeitergespräche, die Vernetzung mit anderen Leitungskräften sowie Teamentwicklungsmaßnahmen. In der Arbeitsteilung zwischen Trägern und Leitungskräften übernahmen die Einrichtungsleitungen den größten Teil der Aufgaben. Einzig bei Personalentscheidungen waren die Träger in nennenswerter Weise involviert (Geiger, 2019, S.50), wobei kommunale und kirchliche Träger stärker in die Personalgewinnung eingebunden waren als freie Träger. Kleine Träger waren häufiger an Mitarbeitergesprächen beteiligt als große Träger (Geiger, 2019, S. 51).

\section{Schlussfolgerungen}

Die Trägerlandschaft ist durch eine große Bandbreite in der Größe, den Rechtsformen und Strukturen der Trägerorganisationen gekennzeichnet. Die Mehrheit der Träger ist für bis zu zehn Einrichtungen verantwortlich. Ehrenamtlich geführte Kita-Träger stellen einen erheblichen
Anteil der Kita-Plätze in Deutschland zur Verfügung. Der Anteil ehrenamtlich geführter Träger ist bei den freien nicht-konfessionellen Trägern am größten, bei den kommunalen Trägern sehr gering. Indessen zeigt sich eine Tendenz, dass freie Träger den kommunalen Einrichtungen hinsichtlich der Professionalität des Personalmanagements leicht überlegen sind - trotz des hohen Anteils Ehrenamtlicher in der Trägerverantwortung. Hinsichtlich der Maßnahmen zur Personalentwicklung zeigt sich, dass Lerngelegenheiten im Prozess der Arbeit - Teamentwicklung, Coaching, Supervision und Fachberatung - ein hoher Stellenwert zugeschrieben wird. Für die eingangs formulierten Vermutungen (BMFSFJ \& JFMK, 2016; Klug \& Kratzmann, 2016), dass nur größere und hauptamtlich geführte Träger eine professionelle Personalarbeit leisten können, finden sich in diesen Daten keine Belege.

\section{Empirische Studie: Personal- entwicklung bei unterschiedlich strukturierten Kita-Trägern}

Wie gestalten unterschiedlich strukturierte Träger von Kindertageseinrichtungen Personalentwicklung in ihren Einrichtungen ? Inwieweit genügen sie dabei den Kriterien professioneller Personalentwicklung?

\section{Analysemodell}

Die Ausgestaltung der Personalentwicklung in Trägerorganisationen von Kindertageseinrichtungen kann in einem Modells zur Beschreibung von Arbeitssystemen theoretisch gefasst werden (vgl. Abb. 1). Das Modell benennt Elemente, welche das Handeln in Arbeitszusammenhängen beeinflussen und stellt sie in ihrem Zusammenspiel dar. Tätigkeiten im Kontext der Personalentwicklung in Kindertageseinrichtungen können damit systematisch erfasst werden (Engestrøm, 2008).

Die Elemente sind:

- Personalverantwortliche als tätige Personen mit individuellen Qualifikationen und Erfahrungen

- die Ziele des Handelns, hier: die professionelle Weiterentwicklung des Personals

- Strategien und Instrumente („Tools“), mit denen die Ziele der Personalentwicklung erreicht werden sollen.

Der Kontext, in welchem das Handeln der Personalverantwortlichen stattfindet, ist gekennzeichnet durch:

- Gesetze und Regeln als normative Rahmenbedingungen des Handelns 
- die „Gemeinschaft" oder fachliche Bezugsgruppe, die u.a. Wissen und fachliche Standards bereitstellt

- die Arbeitsteilung mit Fachleuten oder Institutionen innerhalb und außerhalb der Organisation.

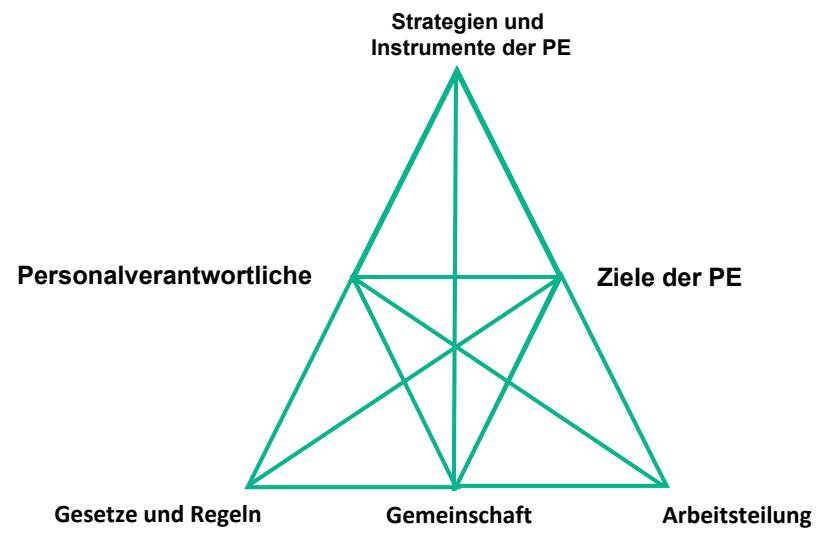

Abbildung 1. Modell zu Arbeitssystemen der Personalentwicklung (PE) nach Engestrøm (2008, S.177).

Die Elemente des Systems sind miteinander verbunden: Die Zielsetzungen und das Handeln der Personalverantwortlichen, wie auch die Wahl der Instrumente und Methoden bewegen sich z.B. im Rahmen der gesetzlichen Vorgaben und sind beeinflusst durch Vereinbarungen über die Arbeitsteilung, beispielsweise mit Leitungskräften oder Fachberatungen. Das Modell ermöglicht u. a. Vergleiche darüber, mit welchen Instrumenten der Personalentwicklung unterschiedliche Träger arbeiten und wie sie die Arbeitsteilung mit den Leitungskräften gestalten.

\section{Empirische Methoden}

Für die Untersuchung von Personalentwicklungsmaßnahmen in Trägerorganisationen wurde ein qualitativ-analytischer Ansatz mit einem Multiple-case-study-design (vgl. Yin, 2009) gewählt. Untersuchungseinheiten (Fälle) sind ausgewählte Trägerorganisationen, in denen die jeweils Personalverantwortlichen als Untersuchungssubjekte befragt wurden. Aufbauend auf dem Modell der Arbeitssysteme wurden die Erhebungsinstrumente konzipiert und Fälle ausgewählt, zu denen empirisches Material gesammelt und die zunächst einzeln ausgewertet wurden. Abschließend wurden die Befunde aus den Fallstudien miteinander verglichen (Abb. 2).

Aus jeder der großen Trägergruppen - kommunale, freigemeinnützige nicht-konfessionelle sowie kirchliche Träger - wurden Organisationen mit mehreren Kindertageseinrichtungen ausgewählt, außerdem eine Elterninitiative mit einer einzigen Einrichtung (vgl. Strehmel \& Overmann, 2018, S.19). Innerhalb der Trägerorganisation wurden zunächst die Vorgesetzten der Leitungskräfte als Personalverantwortliche befragt, außerdem Kita-Leitungen

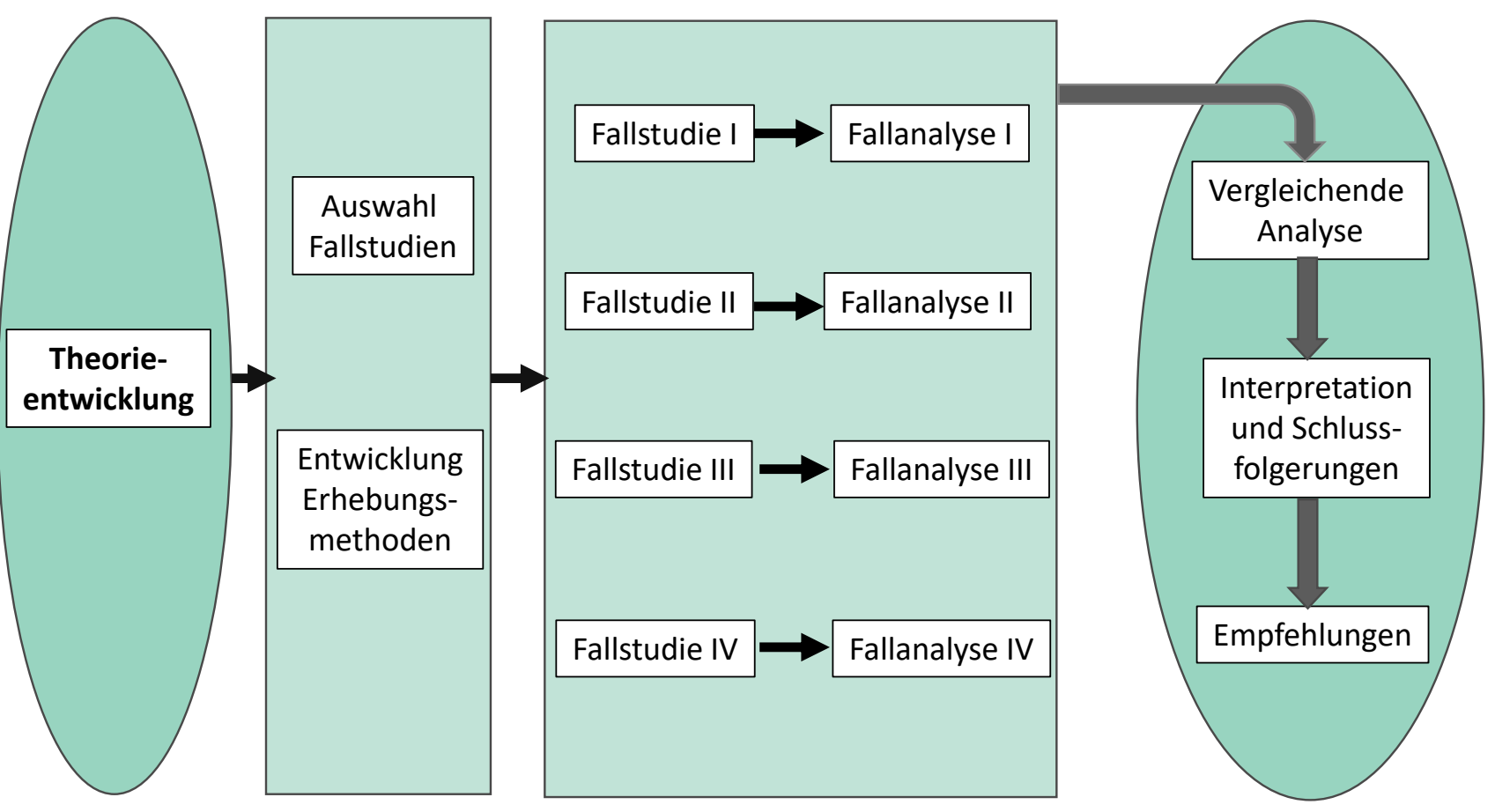

Abbildung 2. Design der Untersuchung: Multiple Fallstudie (vgl. Strehmel \& Overmann, 2018 nach Yin, 2009$).$ 
sowie weitere Personen, die in die Entwicklung des KitaPersonals involviert waren.

\section{Erhebungsmethoden}

Entlang des theoretischen Modells wurden Dokumentenanalysen und qualitative Interviews durchgeführt. In den Dokumentenanalysen wurden Organisationsstrukturen (Arbeitsteilung) sowie normative Rahmenbedingungen (Rechtsform, Leitlinien) der ausgewählten Trägerorganisationen erschlossen. Themen der qualitativen leitfadengestützten Interviews mit den Personalverantwortlichen waren u.a. die Qualifikationen und Berufserfahrungen der Befragten, die jeweiligen Ziele sowie Instrumente und Erfahrungen in verschiedenen Handlungsfeldern des Personalmanagements: Personalgewinnung, Personalführung sowie Personalentwicklung.

\section{Auswertungsmethoden}

Für jede Fallstudie wurden Informationen aus den Dokumenten wie auch aus dem Interviewmaterial in ein auf dem theoretischen Modell basierenden Kategoriensystem eingeordnet. Für verschiedenen Handlungsfelder der Personalentwicklung wurden jeweils Ziele der Befragten, Instrumente (Tools) und Erfahrungen erfasst sowie Organisationsmerkmale (Rechtsform, Größe, Strukturen und Arbeitsteilung) und die Qualifikationen der Befragten („Ordering“, vgl. Miles, Huberman \& Saldaña, 2014). In vergleichenden Tabellen über alle Fallstudien wurden anschließend Gemeinsamkeiten und Unterschiede der Maßnahmen und Strategien herausgearbeitet (Strehmel \& Overmann, 2018, S.105ff.).

\section{Ergebnisse}

Tabelle 2 zeigt die strukturellen Merkmale der Trägerorganisationen. Die Elterninitiative betrieb eine einzige Kindertageseinrichtung, der größte Träger 25 Kitas. Während der kommunale Träger und das kirchliche Unternehmen von hauptamtlichen Führungskräften geleitet wurden, standen Ehrenamtliche an den Spitzen des Sozialunternehmens und der Elterninitiative. Das Sozialunternehmen beschäftigte eine hauptamtliche Geschäftsführung sowie hauptamtliche Fachbereichsleitungen - so auch für die Kindertageseinrichtungen. Im ehrenamtlichen Vorstand der Elterninitiative war eine Person für das Personal zuständig, sie verfügte über eine juristische Qualifikation. Hier war die Leitung durch ein Studium der Kindheitspädagogik hoch qualifiziert.

Während die größeren freien Träger intern wie auch über ihre Dachverbände Fachberatung in Anspruch nehmen konnten, verfügte der kommunale Träger über eine interne Organisationseinheit für Fachberatung. Die Elterninitiative war zugleich Mitglied in einem Spitzenverband der Wohlfahrtspflege wie auch in einem ElterninitiativDachverband. Für Verwaltungsaufgaben verfügten die größeren Träger über eigene Organisationseinheiten, die Elterninitiative beschäftigte eine Verwaltungskraft und einen externen Dienstleister.

Mit welchen Methoden und Instrumenten wurden die Fach- und Leitungskräfte in diesen unterschiedlich strukturierten Trägerorganisationen in ihrer professionellen Weiterentwicklung gefördert? Tabelle 3 gibt einen Überblick über zentrale Maßnahmen der Personalentwicklung bei den untersuchten Trägern entlang der Kriterien professioneller Personalentwicklung.

Fortbildungen wurden bei allen untersuchten Trägern unter Beteiligung der Kita-Teams geplant. Der kirchliche

Tabelle 2. Strukturmerkmale der Trägerorganisationen

\begin{tabular}{|c|c|c|c|c|}
\hline & Kommune & Sozialunternehmen & Kirchlicher Träger & Elterninitiative \\
\hline Zahl der Kitas & 10 & 6 & 25 & 1 \\
\hline Rechtsform & $\begin{array}{l}\text { Körperschaft des } \\
\text { offentlichen Rechts }\end{array}$ & e.V. & gGmbH & e.v. \\
\hline $\begin{array}{l}\text { Haupt-/Ehrenamt } \\
\text { beim Träger }\end{array}$ & Hauptamt & $\begin{array}{l}\text { Ehrenamt im Vorstand, Hauptamt in } \\
\text { Geschäftsführung und Fachbereich }\end{array}$ & Hauptamt & Ehrenamt \\
\hline $\begin{array}{l}\text { Qualifikation der Personal- } \\
\text { verantwortlichen }\end{array}$ & Pädagogik & Pädagogik und BWL & Pädagogik & Jura \\
\hline Verbandszugehörigkeit & - & Wohlfahrtsverband & Wohlfahrtsverband & $\begin{array}{l}\text { Elterninitiativ-Verband \& } \\
\text { Wohlfahrtsverband }\end{array}$ \\
\hline $\begin{array}{l}\text { Fachliches } \\
\text { Unterstützungssystem }\end{array}$ & $\begin{array}{l}\text { Trägerinterne } \\
\text { Fachberatung }\end{array}$ & $\begin{array}{l}\text { Trägerübergreifende } \\
\text { Fachberatung im Verband }\end{array}$ & $\begin{array}{l}\text { Interne \& trägerüber- } \\
\text { greifende Fachberatung }\end{array}$ & $\begin{array}{l}\text { Fachberatung } \\
\text { aus Verbänden }\end{array}$ \\
\hline Verwaltung & Trägerintern & Trägerintern & Trägerintern & $\begin{array}{l}\text { Verwaltungskraft \& } \\
\text { externer Dienstleister }\end{array}$ \\
\hline
\end{tabular}


Tabelle 3. Personalentwicklungsmaßnahmen in den ausgewählten Trägerorganisationen

\begin{tabular}{|c|c|c|c|c|}
\hline & Kommune & Sozialunternehmen & Kirchlicher Träger & Elterninitiative \\
\hline Fortbildungen & $\begin{array}{l}\text { Teamtage; kitaübergreifende } \\
\text { Fortbildungsangebote; } \\
\text { trägerinterne Führungs- } \\
\text { kräfteschulung; }\end{array}$ & $\begin{array}{l}\text { Teamtage; interne Leitungs- } \\
\text { fortbildung; externe Fort- } \\
\text { bildungsangebote; } \\
\text { Steuerung der Teilnahme } \\
\text { nach Organisationszielen }\end{array}$ & $\begin{array}{l}\text { trägerinterne Angebote; } \\
\text { trägerübergreifende } \\
\text { Fortbildung vom Verband }\end{array}$ & $\begin{array}{l}\text { Fortbildungsangebote vom } \\
\text { Verband; } \\
\text { Steuerung über Innovations- } \\
\text { gehalt für die Einrichtung }\end{array}$ \\
\hline $\begin{array}{l}\text { Gelegenheiten } \\
\text { zur Reflexion }\end{array}$ & $\begin{array}{l}\text { Jahresgespräche; } \\
\text { Teambesprechungen; } \\
\text { Arbeitskreise; } \\
\text { Leitungskreis mit Träger }\end{array}$ & $\begin{array}{l}\text { Personalgespräche; } \\
\text { Teambesprechungen; } \\
\text { Arbeitskreise; } \\
\text { Leitungskreis mit Träger }\end{array}$ & $\begin{array}{l}\text { Personalgespräche; Team- } \\
\text { besprechungen; Arbeitskreise; } \\
\text { Leitungskreis mit Träger und } \\
\text { Fachberatung; Klausurtage }\end{array}$ & $\begin{array}{l}\text { Jahresgespräche; } \\
\text { Teambesprechungen; Gesamt- } \\
\text { besprechungen mit Träger; } \\
\text { Teilnahme an Mitglieder- } \\
\text { versammlungen des Vereins }\end{array}$ \\
\hline Fachberatung & trägerintern & extern vom Verband & $\begin{array}{l}\text { trägerintern und extern } \\
\text { vom Verband }\end{array}$ & extern vom Verband \\
\hline $\begin{array}{l}\text { Coaching und } \\
\text { Supervision }\end{array}$ & $\begin{array}{l}\text { internes Coaching durch } \\
\text { den Träger }\end{array}$ & $\begin{array}{l}\text { internes Coaching durch } \\
\text { den Träger }\end{array}$ & $\begin{array}{l}\text { Coachingangebot durch } \\
\text { trägerinterne Fachberatung; } \\
\text { regelhaftes Angebot der } \\
\text { Supervision }\end{array}$ & $\begin{array}{l}\text { regelhafte Verpflichtung } \\
\text { zur Supervision }\end{array}$ \\
\hline
\end{tabular}

Träger entwickelte eigene interne Formate, während die Kommune, das Sozialunternehmen und die Elterninitiative auf externe Angebote zurückgriffen. Im Sozialunternehmen und in der Elterninitiative wurde die Teilnahme an Fortbildungen nach unterschiedlichen Kriterien gesteuert: Im Sozialunternehmen ging es um die Passung von Fortbildungsinteressen zu den Entwicklungszielen der Organisation, während die Leitung der Elterninitiative die pädagogischen Fachkräfte herausforderte, Fortbildungswünsche mit innovativen Ideen für die Einrichtung zu begründen.

Alle Träger wiesen eine ausgeprägte Besprechungskultur auf, in denen die Fach- und Leitungskräfte Gelegenheiten zur Reflexion ihrer Arbeit fanden. Der kommunale Träger orientierte sich in den Jahresgesprächen an den Regeln der Stadtverwaltung, nach denen die jeweils unmittelbaren Vorgesetzten die Gespräche führten. Ähnlich wurde beim kirchlichen Träger verfahren. Das Sozialunternehmen strebte an, mindestens alle zwei Jahre Personalgespräche mit den Beschäftigten zu führen. In der Elterninitiative waren Jahresgespräche etabliert, in die der Personalvorstand involviert war.

Bei allen Trägern konnten die Fach- und Leitungskräfte Fachberatung in Anspruch nehmen, bei der Kommune waren dies trägerinterne Kräfte, bei den anderen Trägern externe Fachberatungen. Bei allen Trägern hatten Fach- und Leitungskräfte Möglichkeiten, kollegiale Beratung oder Coaching in Anspruch zu nehmen. Supervision wurde regelhaft nur beim kirchlichen Träger angeboten bzw. war für das Team der Elterninitiative verbindlich vereinbart.

\section{Diskussion}

Die Personalentwicklungsstrategien der untersuchten Träger erweisen sich weitgehend als professionell im Sinne der zuvor entwickelten Kriterien. Sie weisen eine große Bandbreite auf, doch lassen sich aus den Fallstudien keine systematischen Zusammenhänge zur Größe des Trägers oder der Haupt- oder Ehrenamtlichkeit der Personalverantwortlichen erkennen. Einzelne Bereiche der Personalentwicklung wie etwa regelmäßige Personalgespräche oder die Bereitstellung von Möglichkeiten der Supervision waren unterschiedlich ausgeprägt bzw. befanden sich bei einzelnen Trägern noch in der Entwicklung. Bei allen freien Trägern erwies sich die Zugehörigkeit zu einem Dachverband als wichtige Ressource für den Zugang zu Informationen, Fachberatung und Fortbildung. Nur bei zwei Trägern-dem kirchlichen Unternehmen und der Elterninitiative - war Supervision ein regelmäßiges Angebot zur professionellen Weiterentwicklung des Personals. Coaching war bei den unterschiedlichen Trägern in verschiedenen Formaten möglich, mal durch Vertrauenspersonen beim Träger, mal durch kollegiale Beratung oder freie Fachberatungen.

Die Daten aus Dokumenten und subjektiven Beschreibungen der Personalentwicklung geben noch keinen Aufschluss über die Qualität der Maßnahmen. Weitere Limitierungen dieser Studie liegen in der geringen Zahl der Fälle und der Begrenzung der Interviews auf wenige, für die Personalentwicklung zuständige Personen auf Träger- und Leitungsebene. Möglicherweise gaben die Träger, die sich zur Teilnahme an der Studie bereit erklärten, Beispiele für eine hohe Professionalität und Kreativität in der Ausgestaltung der Personalentwicklung. Die Elterninitiative bestand bereits seit Jahrzehnten und hatte differenzierte Strukturen entwickelt, die hohe pädagogische Qualität auch mit wechselnden ehrenamtlichen Vorständen ermöglichte. Insofern ist die Auswahl der befragten Träger nicht repräsentativ, sie zeigt aber eine Bandbreite auf, die das Potenzial strukturell sehr unterschiedlich aufgestellter Träger hinsichtlich der Personalentwicklung widerspiegelt. 


\section{Fazit}

Die sehr unterschiedlich strukturierten Träger erfüllten die oben genannten Kriterien professioneller Personalentwicklung weitgehend. Kleinere Träger bzw. ehrenamtlich geführte Organisationen fanden dabei teilweise andere Zugänge und Lösungen als größere und hauptamtlich geführte Organisationen. Fehlende fachliche Ressourcen auf Trägerebene wurden bei den kleineren Trägern durch die Vernetzung in Dachverbänden bzw. die Hinzuziehung externer Fachleute kompensiert. Bei allen untersuchten Trägern stellten die Personalverantwortlichen entsprechende Rahmenbedingungen und Unterstützungsstrukturen zur Verfügung.

Zur Steuerung des Systems sollten Maßnahmen zur Qualitätsentwicklung der Träger und zur Unterstützung der Personalentwicklung anschlussfähig sein an die heterogene Trägerlandschaft im System der Kindertagesbetreuung. Für die Professionalität der Personalentwicklung scheinen nicht die Größe des Trägers oder die Hauptamtlichkeit der Verantwortlichen ausschlaggebend zu sein, sondern die Einbindung in Strukturen, aus denen fachliche Ressourcen für die Einrichtungen erschlossen werden können.

Aus diesem Befunden ergeben sich weitere Forschungsdesiderate: Voraussetzung für die Erschließung von Ressourcen aus dem Unterstützungssystem ist der Zugang zu entsprechenden Angeboten und Netzwerken. Inwieweit diese bereits vorhanden sind und genutzt werden, ist bislang kaum erforscht (vgl. dazu BMFSFJ, 2020). Neben strukturellen Fragen bedarf es weiterer Forschung über die notwendigen Kompetenzen der Träger und Möglichkeiten ihrer professionellen Weiterentwicklung. Entsprechende Strukturen könnten dazu beitragen, dass Träger die Personalentwicklung in den Kitas flächendeckend professionell betreiben.

\section{Literatur}

BMFSFJ - Bundesministerium für Familie, Senioren, Frauen und Jugend (2020). Gute-Kita-Bericht 2020. Verfügbar unter: http:// bmfsfj.de/gute-kita-bericht

Beher, K., Krimmer, H., Rauschenbach, T. \& Zimmer, A. (2008). Die vergessene Elite. Führungskräfte in gemeinnützigen Organisationen. Weinheim: Juventa.

BMFSFJ / JFMK - Bundesministerium für Familie, Senioren, Frauen und Jugend \& Jugend- und Familienministerkonferenz (2016): Frühe Bildung weiterentwickeln und finanziell sichern. Zwischenbericht 2016 von Bund und Ländern und Erklärung der Bund-Länder-Konferenz. Berlin. Verfügbar unter: https://www. fruehechancen.de/qualitaet/qualitaetsentwicklungsprozess/ zwischenbericht-2016/

Egert, F., Eckhardt, A. G. \& Fukkink, R. G. (2017). Zentrale Wirkmechanismen von Weiterbildungen zur Qualitätssteigerungen in Kindertageseinrichtungen. Frühe Bildung, 6(2), 58-66.
Engestram, Y. (2008). Entwickelnde Arbeitsforschung: Die Tätigkeitstheorie in der Praxis. Berlin: Lehmanns Media.

Eurofound (2015). Working conditions, training of early childhood care workers and quality of services - A systematic review. Luxembourg: Publications Office of the European Union.

EU - European Union (2011). Competence Requirements in Early Childhood Education and Care. A Study for the European Commission General Directorate for Education and Culture. Final Report. Verfügbar unter: http://www.uel.ac.uk/wwwmedia/schools/ cass/stafffiles/CoRe-final-report-2011.pdf

EU - European Union (2021). Childhood Education and Care: How to recruit, train and motivate well-qualified staff. Luxembourg: European Union.

Falkenhagen, H., Frauendorf, T. \& Bender, N. (2017). Auf Augenhöhe. Leitung von Elterninitiativen in gemeinsamer Verantwortung von Eltern, Erzieherinnen und Erziehern. Gütersloh: Bertelsmann.

Geiger, K. (2019). Personalgewinnung. Personalentwicklung. Personalbindung. Eine bundesweite Befragung von Kindertageseinrichtungen. Weiterbildungsinitiative Frühpädagogische Fachkräfte. WIFF-Studien, Bd. 32. München: DJI.

Kalicki, B. (2003): Qualitätssteuerung durch Rechtsträger. Ergebnisse einer bundesweiten Befragung. In: W. Fthenakis, K. Hansen, P. Oberhuemer \& I. Schreyer (Hrsg.): Träger zeigen Profil. Qualitätshandbuch für Träger von Kindertageseinrichtungen (S.19-29). Weinheim, Basel, Berlin: Beltz.

Klug, W. \& Kratzmann, J. (2016). Erfolgreiches Kita-Management. Unternehmenshandbuch für LeiterInnen und Träger von Kitas. München: Ernst Reinhardt.

Langer, A. (2018). Professionalität und Expertise in der Sozialwirtschaft. In: K. Grunwald \& A. Langer (Hrsg.): Sozialwirtschaft. Handbuch für Wissenschaft und Praxis (S.841-857). BadenBaden: Nomos.

Miles, M. B., Huberman, A. M. \& Saldaña, J. M. (2014). Qualitative Data Analysis: A Methods Sourcebook (3rd edition). London: Sage.

Oberhuemer, P. (2003). TQ-Dimension 4: Personalmanagement. In W. E. Fthenakis, K. Hanssen, P. Oberhuemer \& I. Schreyer (Hrsg.), Träger zeigen Profil. Qualitätshandbuch für Träger von Kindertageseinrichtungen (S.56-61). Weinheim: Beltz.

OECD - Organisation for Economic Cooperation and Development (2018). Engaging Young Children: Lessons from Research about Quality in Early Childhood Education and Care. Starting Strong. Paris: OECD Publishing.

Peucker, C., Pluto, L. \& Santen, E. van (2017). Situation und Perspektiven von Kindertageseinrichtungen. Weinheim: Juventa.

Schreyer, I., Krause, M., Brandl, M. \& Nicko, O. (2014). AQUA - Arbeitsplatz und Qualität in Kitas. Ergebnisse einer bundesweiten Befragung. München. Verfügbar unter: http://www.aqua-studie. de/Dokumente/AQUA_Endbericht.pdf

Sonntag, K. \& Schaper, N. (2016). Berufliche Handlungskompetenz fördern: Wissens- und verhaltensbasierte Verfahren. In K. Sonntag (Hrsg.), Personalentwicklung in Organisationen. Psychologische Grundlagen, Methoden und Strategien (S.369 - 409). Göttingen: Hogrefe.

Statistisches Bundesamt (Destatis) (2020). Statistiken der Kinderund Jugendhilfe. Kinder und tätige Personen in Tageseinrichtungen und in öffentlich geförderter Kindertagespflege am 01.03. 2020. Verfügbar unter: https://www.destatis.de/DE/Themen/ Gesellschaft-Umwelt/Soziales/Kindertagesbetreuung/_inhalt. html\#sprg234640

Strehmel, P. \& Overmann, J. (2018). Personalentwicklung in Kindertageseinrichtungen. Ziele, Strategien und Rolle der Träger. Weiterbildungsinitiative Frühpädagogische Fachkräfte, WiFF-Studien, Bd. 28. München: DJI.

Yin, Robert K. (2009). Case Study Research. Design and Methods. Thousand Oaks: Sage. 
Zapf, D. (2016). Lern- und Entwicklungspotenziale in der Arbeit: Beiträge der Arbeits- und Organisationspsychologie. In K. Sonntag (Hrsg.), Personalentwicklung in Organisationen. Psychologische Grundlagen, Methoden und Strategien (S. 203 - 251). Göttingen: Hogrefe.

\section{Förderung}

Open Access-Veröffentlichung ermöglicht durch die Hochschule für Angewandte Wissenschaften Hamburg.

\section{Petra Strehmel}

Hochschule für Angewandte Wissenschaften Hamburg Department Soziale Arbeit

Alexanderstraße 1

20099 Hamburg

petra.strehmel@haw-hamburg.de 